\title{
Adjunctive Lanicemine (AZD6765) in Patients with Major Depressive Disorder and History of Inadequate Response to Antidepressants: A Randomized, Placebo-Controlled Study
}

\author{
Gerard Sanacora*, , Michael R Johnson ${ }^{2}$, Arif Khan ${ }^{3}$, Sarah D Atkinson ${ }^{4}$, Robert R Riesenberg ${ }^{5}$, \\ Juan P Schronen ${ }^{6}$, Michael A Burke ${ }^{7}$, John M Zajecka ${ }^{8}$, Luis Barra9, Hong-Lin Su ${ }^{10}$, Joel A Posener ${ }^{10}$, \\ Khanh H Bui ${ }^{10,12}$, Michael C Quirk ${ }^{10,12}$, Timothy M Piser ${ }^{10,12}$, Sanjay J Mathew ${ }^{11}$ and Sanjeev Pathak ${ }^{10,12}$ \\ 'Yale University School of Medicine, New Haven, CT, USA; ${ }^{2}$ Sarkis Clinical Trials, Gainesville, FL, USA; ${ }^{3}$ Northwest Clinical Research Center, Bellevue, \\ WA, USA and Department of Psychiatry, Duke University School of Medicine, Durham, NC, USA; ${ }^{4}$ Finger Lakes Clinical Research, Rochester, \\ NY, USA; ${ }^{5}$ Atlanta Center for Medical Research, Atlanta, GA, USA; ${ }^{6}$ Welgemoed Medical Centre, Cape Town, South Africa; ${ }^{7}$ Research Atlanta, \\ Decatur, GA, USA; ${ }^{8}$ Rush University Medical Center, Chicago, IL, USA; ${ }^{9}$ Universidad de Antofagasta, Antofagasta, Chile; ${ }^{10}$ AstraZeneca \\ Pharmaceuticals LP, Wilmington, DE, and Cambridge, MA, USA; "'Baylor College of Medicine, Houston and Michael E. Debakey VA Medical \\ Center, Houston, TX, USA
}

\begin{abstract}
The objective of this study was to investigate the efficacy and safety of adjunctive lanicemine (NMDA channel blocker) in the treatment of major depressive disorder (MDD) over 12 weeks. This phase $\mathrm{llb}$, randomized, parallel-arm, double-blind, placebo-controlled study was conducted at 49 centers in four countries between December 201 I and August 2013 in 302 patients aged I8-70 years, meeting criteria for single episode or recurrent MDD and with a history of inadequate treatment response. Patients were required to be taking an allowed antidepressant for at least four weeks prior to screening. Patients were randomized equally to receive 15 double-blind intravenous infusions of adjunctive lanicemine $50 \mathrm{mg}$, lanicemine $100 \mathrm{mg}$, or saline over a 12 -week course, in addition to ongoing antidepressant. The primary efficacy end point was change in Montgomery-Åsberg Depression Rating Scale (MADRS) total score from baseline to week 6. Secondary efficacy outcome variables included change in MADRS score from baseline to week 12, response and remission rates, and changes in Clinical Global Impression scale, Quick Inventory of Depressive Symptomology Self-Report score, and Sheehan Disability Scale score. Of 302 randomized patients, 240 (79.5\%) completed treatment. Although lanicemine was generally well tolerated, neither dose was superior to placebo in reducing depressive symptoms on the primary end point or any secondary measures. There was no significant difference between lanicemine and placebo treatment on any outcome measures related to MDD. Post hoc analyses were performed to explore the possible effects of trial design and patient characteristics in accounting for the contrasting results with a previously reported trial.

Neuropsychopharmacology (2017) 42, 844-853; doi:I0.I 038/npp.20 I6.224; published online 2 November 2016
\end{abstract}

\section{INTRODUCTION}

A growing number of reports demonstrate the ability of ketamine to induce robust antidepressant effects and suggest that drugs targeting the $\mathrm{N}$-methyl-D-aspartate receptor (NMDAR) may provide a novel class of antidepressant drug that addresses the unmet demands for rapid-acting antidepressants and efficacy in treatment-resistant depression (McGirr et al, 2015; Newport et al, 2015). However, there are several factors such as functional unblinding and acute physiological and psychological effects that create challenges

*Correspondence: Dr G Sanacora, Clinical Neuroscience Research Unit, Yale University School of Medicine, 100 York St, Suite 2), New Haven, CT 065 I I, USA, Tel: + I 203974 7535, Fax: + I 203974 7662, E-mail: gerard.sanacora@yale.edu

${ }^{12}$ Former employee.

Received 4 May 2016; revised 17 August 2016; accepted 8 September 2016; accepted article preview online 29 September 2016 to the interpretation of the findings, and limit the use of ketamine in clinical settings (Sanacora and Schatzberg, 2015; Schatzberg, 2014). These factors motivate the search for novel compounds that possess the critical antidepressant properties of ketamine without the same magnitude of unwanted effects on cognition, perception, and cardiovascular function.

Lanicemine (AZD6765) is a low-trapping, parenterally administered NMDA channel blocker sharing many of the same pharmacological effects as ketamine at the NMDAR. (Emnett et al, 2013; Sanacora et al, 2014) However, at doses shown to have similar effects on absolute gamma EEG magnitude in the frontal cortex as those seen with the commonly used intravenous infusion dose of ketamine $(0.5 \mathrm{mg} / \mathrm{kg}$ over $40 \mathrm{~min})$, the effects of lanicemine on perception and cognition are greatly reduced (Sanacora et al, 2014). Two small proof-of-concept studies found preliminary evidence suggesting that a single dose of 
lanicemine was associated with a rapid but short-lived antidepressant response, albeit less robust than that previously reported with ketamine, in the absence of clinically meaningful effects on cognition and perception (Sanacora et al, 2014; Zarate et al, 2013). More recently, a phase IIb study of 152 patients (Study 9) reported that lanicemine (100 or $150 \mathrm{mg}$, three intravenous infusions/week for 3 weeks, adjunctive to one ongoing antidepressant) was associated with a significant improvement of depressive symptoms in patients with treatment-resistant major depressive disorder (MDD) over that seen with placebo (saline) infusions at the predetermined 3-week time point (Sanacora et al, 2014).

The current phase IIb study (Study 31) was designed to investigate the efficacy, safety, and tolerability of adjunctive lanicemine (50 or $100 \mathrm{mg} /$ infusion) repeatedly administered at decreasing frequency over 12 weeks in patients with MDD, with the primary outcome measure of interest being the change in Montgomery-Åsberg Depression Rating Scale (MADRS) total score from baseline to week 6. The two doses were chosen because the 100-mg lanicemine dose was associated with a better outcome in nearly all efficacy measures in Study 9, and because emerging evidence suggests the possibility of an inverted U-type dose-response curve with other NMDAR-targeting drugs (Burgdorf et al, 2013; Chowdhury et al, 2016; Li et al, 2010). The 6-week end point was considered the most relevant to assess short-term antidepressant effects. This manuscript reports the primary efficacy results from this study together with safety assessments. Additional post hoc analyses attempting to investigate the differences in outcome between the two phase IIb studies (Studies 9 and 31) are also presented, as they could be highly relevant to optimization of future study designs attempting to evaluate the antidepressant efficacy of this unique class of drugs.

\section{MATERIALS AND METHODS}

\section{Study Design}

This was an AstraZeneca-sponsored phase IIb, randomized, parallel-arm, double-blind, placebo-controlled study conducted at 49 centers in Chile, Slovakia, South Africa, and the United States between 16 December 2011 and 26 August 2013. The study was performed in accordance with the ethical principles originating in the Declaration of Helsinki and consistent with the International Conference on Harmonisation/Good Clinical Practice. All patients provided written, informed consent and were free to discontinue the study at any time. The study was registered with clinicaltrials. gov (NCT01482221).

The study consisted of three phases: an evaluation and washout phase lasting up to 6 weeks, a randomized adjunctive treatment phase lasting 12 weeks, and a 2-week post-treatment follow-up phase (changed from an 8-week follow-up in a protocol amendment due to difficulties in retaining subjects, now off of all study medications, for the extended period of time).

\section{Evaluation and Washout Phase}

Male or female outpatients aged 18-70 years meeting criteria for single episode or recurrent MDD based on Diagnostic and Statistical Manual of Mental Disorders, 4th Edition, Text Revision (DSM-IV-TR), confirmed by the Mini International Neuropsychiatric Interview, and with a history of inadequate response to three or more antidepressants (including one antidepressant in the current depressive episode) confirmed by the Antidepressant Treatment History Form (Oquendo et al, 2003) were eligible for participation. Inadequate response to previous trials was defined as persistent symptoms that, as judged by the investigator, continued to meet diagnostic criteria for MDD according to DSM-IV-TR. Patients were required to be taking an allowed antidepressant treatment for at least 4 weeks prior to screening at a prespecified dose range. A list of allowed concomitant antidepressants along with minimum dose and duration of treatment is provided in Supplementary eTable S1. Full descriptions of inclusion and exclusion criteria are provided in the Supplementary Text.

\section{Randomized Treatment Phase and Follow-Up}

Subjects entering the study drug treatment phase were randomized in balanced blocks equally (1:1:1 ratio), using a unique randomization code generated via Interactive Voice Response System, to receive a 12-week course of doubleblind, intravenous infusions of lanicemine $50 \mathrm{mg}$, lanicemine $100 \mathrm{mg}$, or placebo (saline). The investigator, patient, and study staff were all blinded. Packaging and labeling of study medications could not be used by the investigators or subjects to determine randomization assignment.

Study drug was infused three times a week on nonconsecutive days in weeks 1 to 3 , once a week in weeks 4 to 6 , and once every 2 weeks in weeks 7 to 12 (Figure 1), based on the findings of study 9 using thrice weekly dosing for 3 weeks and attempting to explore the effects of a tapered dosing frequency for longer-term care. Study medication was infused in a total volume of $100 \mathrm{ml}$ given over $1 \mathrm{~h}$ $(0.833 \mathrm{mg} / \mathrm{min}$ for the lanicemine $50-\mathrm{mg}$ dose; $1.667 \mathrm{mg} /$ min for the 100-mg dose). After the first infusion, patients remained on-site for at least $3 \mathrm{~h}$. Starting from the second infusion, patients remained on-site for at least $1 \mathrm{~h}$ after the end of infusion. Prior to release from the site, patients had to demonstrate stable vital signs and were questioned about any adverse events (AEs) being experienced.

Patients were scheduled for a follow-up visit 14 days ( \pm 4 days) from last dosing (week 14) to collect laboratory measurements and other assessments.

\section{Outcome Measures}

The primary planned efficacy outcome was change in MADRS total score from baseline to week 6 in the adjunctive lanicemine-treated groups (50 or $100 \mathrm{mg} /$ infusion) compared with the adjunctive placebo group.

Secondary efficacy outcome variables were: change in MADRS total score from baseline (except week 6); rate of response (ie, $\geqslant 50 \%$ reduction from baseline in MADRS total score); rate of remission (ie, MADRS total score $\leqslant 10$ ); and changes in severity of depressive symptoms measured by CGI-S scale and Clinical Global Impression-Improvement (CGI-I) scale (GCI response defined as 'very much improved' or 'much improved') (Guy, 1976), severity of depressive symptoms measured by QIDS-SR-16 total score, 


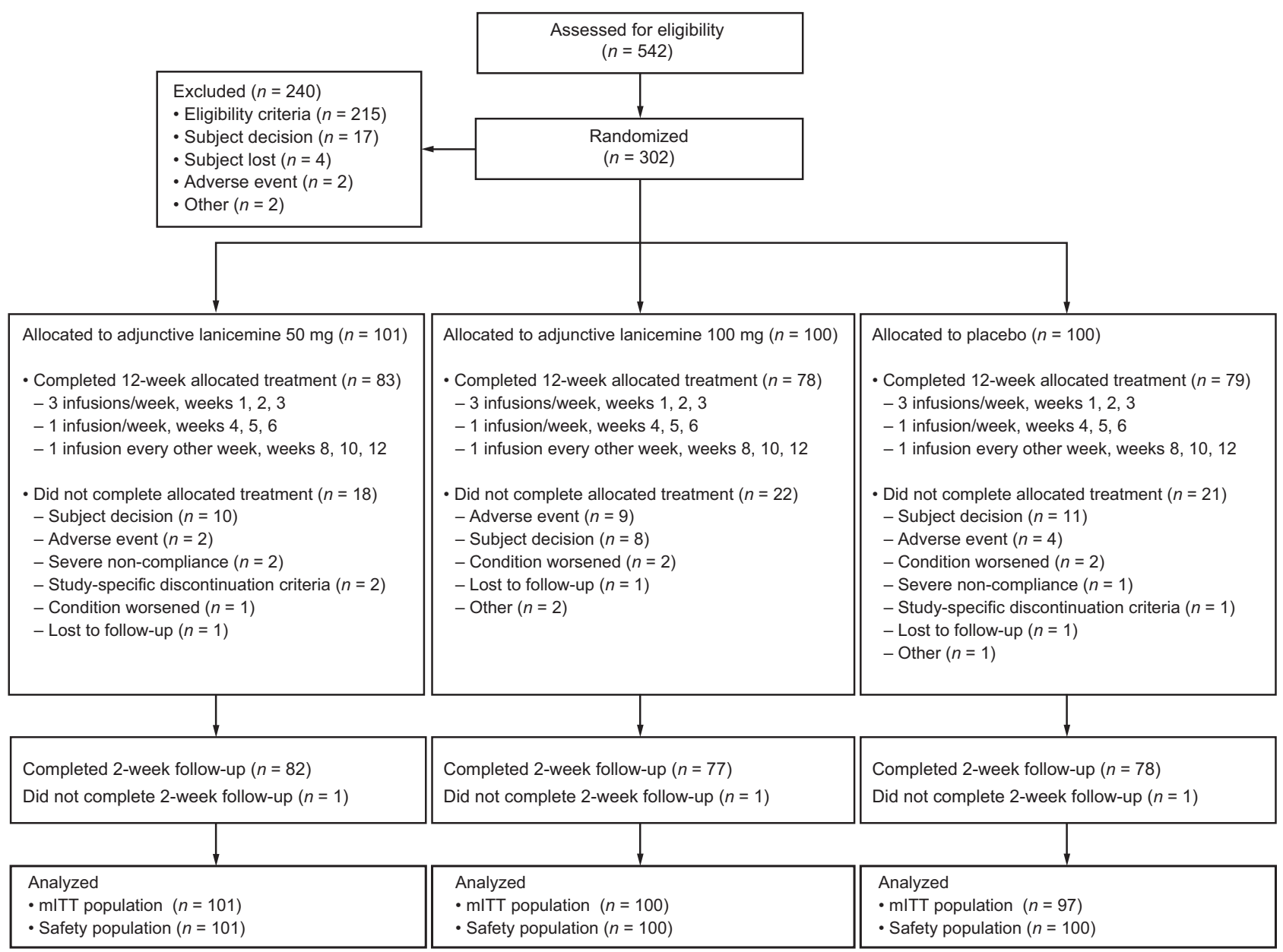

Figure I Study design and disposition.

and functional impairment measured by Sheehan Disability Scale (SDS) total score.

Safety variables including AEs (coded using the Medical Dictionary for Drug Regulatory Affairs (MedDRA), version 16.0), vital signs, electrocardiogram (ECG), laboratory measures, CogState Battery tests (a series of four computerized neurocognitive tests: Detection, Identification, One-card Learning, and One-back tasks; CogState, New Haven, CT), and Clinician Administered Dissociative States Scale (CADSS) total score (Bremner et al, 1998) were examined to compare lanicemine $v s$ placebo groups. The schedules of safety assessments are included in Supplementary eTable S2.

Treatment compliance was calculated as the number of infusions received divided by the number prescribed.

\section{Post Hoc Analyses}

As there was significant interest in exploring factors that could be related to outcome differences between the previous phase IIb study with lanicemine (Study 9) (Sanacora et al, 2014) and the current study (Study 31), we compared these studies on protocol design, demographic and descriptive statistics, as well as change in MADRS score and the percent response at the week- 4 time point (the only time point collected consistently across both protocols). Details of these analyses are included in the Supplementary Text File.

\section{Statistical Methods}

Efficacy analyses were based on the modified intent-to-treat (mITT) analysis set, including all randomized patients who received at least one dose of study drug and who had at least one post-baseline MADRS score. Safety and tolerability assessments were based on the safety analysis set, including all randomized patients who received study drug and for whom post-dose data were available.

The primary efficacy variable, change from baseline to week 6 in the MADRS total score, was analyzed using a mixed-model repeated measures (MMRM) analysis of all post-baseline MADRS total scores through the end of the study (week 14). The model included treatment, visit, treatment by visit interaction, and baseline MADRS total score by visit as fixed effects, and site as a random effect. Restricted maximum likelihood with an unstructured variance-covariance matrix was used for estimation in the MMRM analysis. Each lanicemine dose was compared with placebo.

The same MMRM approach was used for other continuous secondary efficacy variables. Binary secondary efficacy 
variables with multiple post-baseline assessments (response, remission, and CGI-I response) were analyzed using a generalized linear model of the repeated measures. A logit link function was used and the statistical inferences were based on generalized estimating equations. The independent variables were treatment, visit, treatment by visit interaction term, and a baseline measurement related to the dependent analysis variable. For response and remission, baseline MADRS total score was used as the baseline measurement; for CGI-I response, baseline CGI-S score was used. Safety outcomes were presented using appropriate descriptive statistics.

Post hoc statistical analyses for Study 31 were informed by review of the antidepressant literature and the assessment of differences in study design between Studies 9 and 31. The analyses were performed similarly to the prespecified analyses except subgroups were identified in a post hoc manner. Comparison of efficacy end points between Studies 9 and 31 were extracted directly from the corresponding study reports for informational purposes only and had no statistical validity.

The sample size calculation in Study 31 was planned to ensure $90 \%$ power to show that at least one of the two lanicemine doses was statistically significantly superior to placebo for the primary outcome variable at week 6, assuming a difference of 5.5 units of lanicemine from placebo and an SD of 10 for the change in MADRS total score from baseline to week 6. Based on the Bonferroni multiplicity adjustment, the one-sided significance level was planned at 0.0125 .

\section{RESULTS}

\section{Participant Characteristics}

Of 542 patients enrolled in the study, 302 were randomized, $240(79.5 \%)$ completed treatment, and 238 (78.8\%) completed treatment plus follow-up (Figure 1). The most common reason for non-randomization (in 240 patients) was eligibility criteria not fulfilled ( $n=215,89.6 \%)$. The most common reason for discontinuation of treatment (in 61 patients) was subject decision $(n=29,9.6 \%$ of total population: $9.9 \%$ of adjunctive lanicemine $50 \mathrm{mg}, 7.9 \%$ of adjunctive lanicemine $100 \mathrm{mg}$, and $11.0 \%$ of adjunctive placebo groups). Baseline demographic and disease characteristics were similar between the treatment groups (Table 1). Baseline mean (SD) MADRS scores were similar between the 97 subjects randomized to placebo (35.64 (4.84)), 101 subjects randomized to $50 \mathrm{mg}$ lanicemine (36.55 (4.67)), and 100 subjects randomized to $100 \mathrm{mg}$ lanicemine $(36.02(4.74))$ in ITT analysis.

The majority of patients $(92.7 \%)$ had an acceptable overall compliance rate (ie, $\geqslant 80 \%$ to $\leqslant 100 \%$ ). The duration of exposure (from treatment start to study completion or early discontinuation) was similar in all groups: mean 74.3 (SD 20.7) days in the lanicemine 50-mg, 70.3 (23.2) days in the lanicemine 100-mg, and 71.0 (22.9) days in the placebo group.

\section{Efficacy Outcomes}

The primary planned outcome measure, the least-squares (LS) mean (standard error, SE) change in MADRS total score at week 6 , was $-14.37(1.24)$ in the adjunctive lanicemine $50-\mathrm{mg},-14.40(1.24)$ in the adjunctive lanicemine 100-mg, and -13.18 (1.27) in the adjunctive placebo group. The difference between lanicemine and placebo groups was nonsignificant: $-1.18(P=0.485)$ for lanicemine $50 \mathrm{mg}$ and $-1.21(P=0.476)$ for lanicemine $100 \mathrm{mg}$ (mITT analysis set). Lanicemine groups also did not differ significantly from placebo in any secondary efficacy variables at week 6 (Table 2). The LS mean (SE) change in MADRS total score at week 12 was -15.97 (1.31) in the lanicemine $50-\mathrm{mg}$, -13.03 (1.33) in the lanicemine $100-\mathrm{mg}$, and - 13.92 (1.35) in the placebo groups. Again, the differences in LS mean change between lanicemine and placebo groups were nonsignificant: $-2.05(P=0.259)$ for the lanicemine $50-\mathrm{mg}$ group and $0.88(P=0.630)$ for the $100-\mathrm{mg}$ group (Figure 2$)$. The difference in LS mean change in MADRS total score between lanicemine and placebo groups was non-significant at every study visit (Figure 2).

\section{Safety Outcomes}

At least one $\mathrm{AE}$ was reported by $77.1 \%$ patients in the lanicemine groups and $70.0 \%$ in the placebo group, with most AEs of mild or moderate intensity (Supplementary eTable S3). Dizziness was the most common AE (mild/ moderate in severity in all cases). Six patients (3.0\%) who received lanicemine ( $n=2$ patients in the $50-\mathrm{mg}$ group and $n=4$ in the $100-\mathrm{mg}$ group) and four patients (4.0\%) in the placebo group experienced a serious AE (SAE). The SAEs included anemia and asthma in the lanicemine $50 \mathrm{mg}$ group; hepatitis C, depressive symptom, intentional drug misuse, and worsening depression in the lanicemine 100-mg group; and chest pain, acute alcohol intoxication/alcohol poisoning, increased risk of suicidal ideation, and suicidal ideation in the placebo group.

More patients discontinued treatment because of an AE in the lanicemine $100-\mathrm{mg}$ group $(9.0 \%, n=9)$ than in the lanicemine $50-\mathrm{mg}(2.0 \%, n=2)$ or placebo groups $(4.0 \%$, $n=4)$. Reasons for discontinuation included rash $(n=2)$ in the lanicemine 50-mg group; rash $(n=2)$, vision blurred, peripheral edema, bronchitis, clumsiness, sedation, anxiety, depressive symptoms, intentional drug misuse, major depression, and suicidal ideation $(n=1$, each) in the lanicemine 100-mg group; and gastritis, eosinophil count increased, suicidal ideation, and rash $(n=1$, each) in the placebo group. The numbers of AEs associated with psychotomimetic or dissociative symptoms were low for all treatment groups. Proportions of patients experiencing psychotomimetic AEs during randomized treatment (including bradyphrenia, sensory disturbance, dysarthria, and visual hallucination) were $4.0 \%(n=4)$ in the lanicemine $50-\mathrm{mg}$, $2.0 \%(n=2)$ in the lanicemine $100-\mathrm{mg}$, and $0.0 \%$ in the placebo groups. Of these events, only one (visual hallucination, lanicemine 50-mg group) was clinically considered a true psychotomimetic AE, that is, mimicking psychosis, and this did not lead to study discontinuation. The proportion of patients experiencing a dissociative $\mathrm{AE}$ during treatment (including dissociation, depersonalization, and illusion) was higher in the lanicemine $100-\mathrm{mg}$ group $(11.0 \%, n=11)$ than in the lanicemine $50-\mathrm{mg}(4.0 \%, n=4)$ or placebo groups $(4.0 \%, n=4)$. All dissociative AEs were mild and none led to treatment discontinuation. 
Lanicemine trial in major depressive disorder

G Sanacora et al

Table I Demographic Characteristics and Disease Characteristics (All Randomized Patients) in Study 3 I

\begin{tabular}{|c|c|c|c|c|c|c|c|c|}
\hline \multirow[t]{2}{*}{ Demographic and disease characteristics } & \multicolumn{2}{|c|}{ Placebo $(n=100)$} & \multicolumn{2}{|c|}{$\begin{array}{l}\text { Lanicemine } 50 \mathrm{mg} \\
\quad(n=\mid 0 I)\end{array}$} & \multicolumn{2}{|c|}{$\begin{array}{c}\text { Lanicemine } \\
100 \mathrm{mg}(n=101)\end{array}$} & \multicolumn{2}{|c|}{ Total $^{\mathrm{a}}(\mathbf{N}=302)$} \\
\hline & Mean & (SD) & Mean & (SD) & Mean & (SD) & Mean & (SD) \\
\hline \multirow[t]{2}{*}{ Age, years ${ }^{b}$} & 49.5 & $(11.12)$ & 47.7 & $(11.19)$ & 47.5 & $(11.89)$ & 48.2 & $(11.40)$ \\
\hline & $n$ & (\%) & $n$ & (\%) & $n$ & (\%) & $n$ & (\%) \\
\hline \multicolumn{9}{|l|}{ Age group, years ${ }^{b}$} \\
\hline $18-39$ & 19 & $(19.0)$ & 24 & $(23.8)$ & 22 & $(21.8)$ & 65 & $(21.5)$ \\
\hline $40-64$ & 74 & $(74.0)$ & 74 & $(73.3)$ & 74 & $(73.3)$ & 222 & (73.5) \\
\hline$\geqslant 65$ & 7 & $(7.0)$ & 3 & $(3.0)$ & 5 & $(5.0)$ & 15 & $(5.0)$ \\
\hline \multicolumn{9}{|l|}{ Sex } \\
\hline Male & 35 & $(35.0)$ & 39 & $(38.6)$ & 31 & $(30.7)$ & 105 & (34.8) \\
\hline Female & 65 & $(65.0)$ & 62 & $(61.4)$ & 70 & $(69.3)$ & 197 & $(65.2)$ \\
\hline \multicolumn{9}{|l|}{ Race } \\
\hline White & 91 & $(91.0)$ & 91 & $(90.1)$ & 87 & $(86.1)$ & 269 & $(89.1)$ \\
\hline Black or African American & 6 & $(6.0)$ & 8 & $(7.9)$ & 11 & $(10.9)$ & 25 & $(8.3)$ \\
\hline Asian & I & $(1.0)$ & 0 & $(0)$ & 3 & $(3.0)$ & 4 & $(1.3)$ \\
\hline Native Hawaiian or other Pacific Islander & 0 & $(0)$ & 0 & $(0)$ & 0 & $(0)$ & 0 & \\
\hline American Indian or Alaska Native & 0 & $(0)$ & 0 & $(0)$ & 0 & $(0)$ & 0 & \\
\hline Other & 2 & $(2.0)$ & 2 & $(2.0)$ & 0 & $(0)$ & 4 & $(1.3)$ \\
\hline \multicolumn{9}{|l|}{ Ethnic group } \\
\hline Hispanic or Latino & 14 & $(14.0)$ & 14 & $(13.9)$ & 7 & $(6.9)$ & 35 & $(11.6)$ \\
\hline Not Hispanic or Latino & 86 & $(86.0)$ & 87 & $(86.1)$ & 93 & $(92.1)$ & 266 & $(88.1)$ \\
\hline Unknown & 0 & $(0)$ & 0 & $(0)$ & । & $(1.0)$ & । & $(0.3)$ \\
\hline \multicolumn{9}{|l|}{ Previous depressed episodes over lifetime } \\
\hline 0 & 11 & $(\mid 1.0)$ & 6 & $(5.9)$ & 4 & $(4.0)$ & 21 & $(7.0)$ \\
\hline 1 & 10 & $(10.0)$ & 11 & $(10.9)$ & 11 & $(10.9)$ & 32 & $(10.6)$ \\
\hline 2 & 12 & $(12.0)$ & 8 & $(7.9)$ & 14 & $(13.9)$ & 34 & $(11.3)$ \\
\hline$\geqslant 3$ & 65 & $(65.0)$ & 75 & $(74.3)$ & 70 & $(69.3)$ & 210 & $(69.5)$ \\
\hline Generalized anxiety disorder & 14 & $(14.0)$ & 16 & $(15.8)$ & 7 & $(6.9)$ & 37 & $(12.3)$ \\
\hline \multicolumn{9}{|l|}{ Number of patients with combination therapy } \\
\hline Yes & 68 & $(68.0)$ & 72 & $(7 \mid .3)$ & 74 & $(73.3)$ & 214 & $(70.9)$ \\
\hline No & 32 & $(32.0)$ & 29 & $(28.7)$ & 27 & $(26.7)$ & 88 & $(29.1)$ \\
\hline \multicolumn{9}{|l|}{ Number of patients with concomitant psychotropics } \\
\hline With I concomitant psychotropic & 28 & $(28.0)$ & 43 & $(42.6)$ & 31 & $(30.7)$ & 102 & $(33.8)$ \\
\hline With 2 concomitant psychotropics & 9 & $(9.0)$ & 5 & $(5.0)$ & 7 & $(6.9)$ & 21 & $(7.0)$ \\
\hline With 3 concomitant psychotropics & 2 & $(2.0)$ & 0 & $(0)$ & 3 & $(3.0)$ & 5 & $(1.7)$ \\
\hline With 4 or more concomitant psychotropics & 0 & & 0 & $(0)$ & । & $(1.0)$ & I & $(0.3)$ \\
\hline \multicolumn{9}{|c|}{ Number of patients with inadequate responses during the current episode ${ }^{c}$} \\
\hline । & 25 & $(25.0)$ & 16 & $(15.8)$ & 20 & $(19.8)$ & 61 & $(20.2)$ \\
\hline 2 & 18 & $(\mid 8.0)$ & 18 & $(17.8)$ & 15 & $(14.9)$ & 51 & $(16.9)$ \\
\hline 3 & 19 & $(19.0)$ & 23 & $(22.8)$ & 22 & $(21.8)$ & 64 & $(21.2)$ \\
\hline$\geqslant 4$ & 24 & $(24.0)$ & 32 & $(31.7)$ & 27 & $(26.7)$ & 83 & (27.5) \\
\hline
\end{tabular}


Table I Continued

\begin{tabular}{|c|c|c|c|c|c|c|c|c|}
\hline \multirow[t]{2}{*}{ Demographic and disease characteristics } & \multicolumn{2}{|c|}{ Placebo $(n=100)$} & \multicolumn{2}{|c|}{$\begin{array}{l}\text { Lanicemine } 50 \mathrm{mg} \\
\qquad(n=10 \mathrm{I})\end{array}$} & \multicolumn{2}{|c|}{$\begin{array}{c}\text { Lanicemine } \\
100 \mathrm{mg}(n=101)\end{array}$} & \multicolumn{2}{|c|}{ Total $^{\mathbf{a}}(\mathbf{N}=302)$} \\
\hline & Mean & (SD) & Mean & (SD) & Mean & (SD) & Mean & (SD) \\
\hline Baseline MADRS score sample size $^{d}$ & 97 & & 101 & & 100 & & 298 & \\
\hline Baseline MADRS score & 35.64 & $(4.84)$ & 36.55 & $(4.67)$ & 36.02 & $(4.74)$ & & \\
\hline
\end{tabular}

Abbreviation: MADRS, Montgomery-Åsberg Depression Rating Scale.

Note: The percentages are based on the number of patients in each treatment group. Missing data are not included.

'The 'Total' column summarizes all treatment groups.

${ }^{\mathrm{b}} \mathrm{Age}$ is calculated as the rounded down integer value in years of ((date of consent - date of birth)/365.25).

'The number of inadequate responses is calculated the same way as that for during their lifetime (including reliability of source and duration criteria), except the procedure start date is on or after date of onset of present MDD episode.

${ }^{\mathrm{d}} \mathrm{mlTT}$ analysis set.

Table 2 Secondary Efficacy Variables: Assessments at Week 6

\begin{tabular}{|c|c|c|c|}
\hline Response rate (\%) & $39.0 \%$ & $36.0 \%$ & $44.0 \%$ \\
\hline Remission rate (\%) & $18.3 \%$ & $23.3 \%$ & $23.8 \%$ \\
\hline Adjusted OR vs placebo (mean, SE) & & $1.42(0.38)(P=0.357)$ & $1.33(0.39)(P=0.463)$ \\
\hline CGI-S score change (LS mean, SE) & $-1.4(0.16)$ & $-1.5(0.16)$ & $-1.5(0.16)$ \\
\hline CGI-I response (\%) & $37.8 \%$ & $51.2 \%$ & $47.6 \%$ \\
\hline Adjusted OR vs placebo (mean, SE) & & $1.74(0.30)(P=0.067)$ & $1.43(0.30)(P=0.230)$ \\
\hline QIDS-SR-16 score change (LS mean, SE) & $-8.1(0.7 I)$ & $-8.7(0.69)$ & $-7.9(0.70)$ \\
\hline Difference in LS mean (SE) change vs placebo & & $-0.6(0.87)(P=0.505)$ & $0.2(0.88)(P=0.842)$ \\
\hline SDS score (LS mean, SE) & $-6.9 \mid(0.99)$ & $-7.08(0.96)$ & $-6.90(0.98)$ \\
\hline
\end{tabular}

Abbreviations: CGI I, Clinical Global Impression-Improvement; CGI-S, Global Impression-Severity of Illness; LS, least squares; OR, odds ratio; QIDS-SR-I6, Quick Inventory of Depressive Symptomology Self-Report 16 item scale.

Treatment with 50- or 100-mg lanicemine was not associated with any decline in psychomotor function, attention, working memory, learning, or general cognitive function in CogState test battery tasks performed prior to infusion on study visit days (Supplementary eFigure S1). There was some evidence that lanicemine was associated with a modest increase in dissociative symptoms in a relatively small subgroup of patients, assessed by the CADSS at the time of infusion completion. A greater proportion of patients in the lanicemine $50-\mathrm{mg}(13.2 \%, n=12)$ and lanicemine 100 -mg groups $(14.9 \%, n=13)$ had CADSS total scores in the medium score (ie, 3-10) range compared with the placebo group $(7.0 \%, n=6)$ at week 6. A greater proportion of patients in the lanicemine 50-mg (1.1\%, $n=1)$ and lanicemine $100-\mathrm{mg}$ groups $(1.1 \%, n=1)$ had a CADSS total score in the high score (ie, 11 to 25 ) range compared with the placebo group (no patients) at week 6 .

There were no clinically meaningful differences between lanicemine and placebo groups in clinical laboratory test values (clinical chemistry, hematology, and urinalysis) during the randomized treatment and follow-up periods. Lanicemine infusion was associated with modest, transient increases in supine systolic blood pressure (SBP) and diastolic blood pressure (DBP), which were more prominent with the 100-mg than 50-mg dose (eg, change in mean supine DBP at the end of infusion $v$ s preinfusion at week 6 was approximately $0.8,2.2$, and $0.5 \mathrm{~mm} \mathrm{Hg}$ in the lanicemine 50-mg, lanicemine 100-mg, and placebo groups, respectively). Changes in blood pressure appeared to resolve spontaneously during study (eg, change in mean supine DBP at last assessment on treatment $v s$ baseline was $0.0,0.5$, and $-0.5 \mathrm{~mm} \mathrm{Hg}$ in the lanicemine $50-\mathrm{mg}$, lanicemine 100-mg, and placebo groups, respectively). A numerically greater proportion of patients in the lanicemine $50-\mathrm{mg}$ (43.6\%, $n=44)$ and lanicemine $100-\mathrm{mg}$ groups $(40.0 \%$, $n=40)$ had $\geqslant 10 \mathrm{~mm} \mathrm{Hg}$ decreases from supine to standing DBP after at least 3 min compared with the placebo group $(28.9 \%, n=28)$ at any time point in the study. There were no notable differences between the lanicemine and placebo groups in ECG parameters during the randomized treatment and follow-up periods.

\section{Post Hoc Analyses}

Several differences were noted in the protocol design between Study 31 and Study 9, as described in 


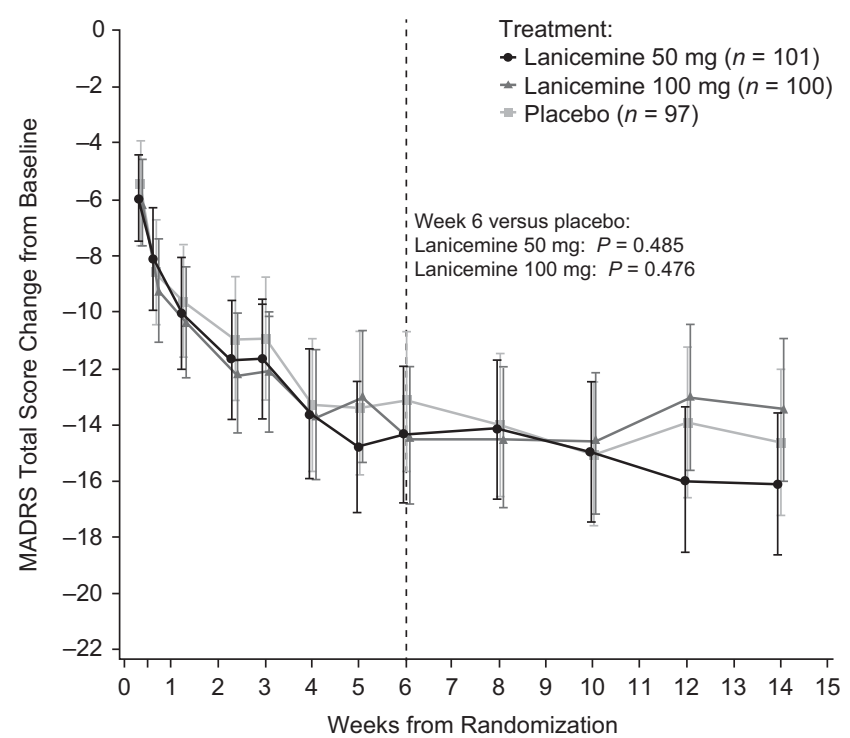

Figure 2 LS mean and $95 \% \mathrm{Cl}$ of change in MADRS total score from baseline (for each visit by treatment), MMRM, OC (mITT analysis set). Abbreviation: LS, least-squares; MADRS, Montgomery-Åsberg Depression Rating Scale; MMRM, mixed model repeated measures; mITT, modified intent-to-treat; OC, observed cases.

Supplementary eTable S4. Comparing the change in MADRS score from baseline to week 4 (the single shared time point of the studies), Studies 9 and 31 showed similar MADRS score changes in the adjunctive lanicemine 100-mg groups (dose common to both studies) from baseline (-13.3 vs -13.8 , respectively). However, the Study 31 adjunctive placebo group showed a greater reduction in MADRS score than the Study 9 placebo group $(-13.9$ vs -9.1$)$ (Figure 3). Variance (SD) in MADRS change from baseline was also higher in Study 31. The 13.9 point change in mean MADRS score in the placebo group was outside the $95 \%$ CI $(-11.92$ to -6.28$)$ that was determined for placebo response in Study 9 at week 4. Likewise, response rates at week 4 were similar in the lanicemine 100-mg groups in Study 31 and Study 9 (36.7\% vs $37.3 \%)$, but higher response rates were observed for adjunctive placebo in Study 31 than Study $9(31.4 \%$ vs 20.0\%).

Considering the differences in study protocols identified from the comparison of study designs (Supplementary eTable S4), we explored the impact of several variables that could have contributed to the differences in outcome measures obtained at the week 4 time point on the Study 31 data set. Greater depressive symptom severity at baseline (based on MADRS score tertile) was associated with larger lanicemine-placebo score differences, especially for the 100mg lanicemine dose. Moreover, the largest placebo response was seen in patients in the lowest MADRS tertile, that is, MADRS score change: -15.2 for patients with baseline MADRS score $<34$, vs -11.0 and -13.3 for baseline MADRS scores $34-<38$ and $\geqslant 38$, respectively (Supplementary eFigure S2). Subjects experiencing baseline suicidal thoughts, assessed by MADRS item 10 score $\geqslant 2$, also showed a trend for a numerically superior response to lanicemine over placebo. In these patients, mean (SD) MADRS score change at week 6 was -13.0 (12.6), - 16.5
(14.6), and $-9.2(11.2)$ in the lanicemine 50-mg $(n=48)$, $100-\mathrm{mg}(n=46)$, and placebo groups $(n=37)$, respectively. A similar trend on outcome was noted in relation to antipsychotic medication use: LS mean (SE) MADRS score change from baseline at week 6 in patients concomitantly taking antipsychotic medications was - $11.56(2.35),-14.72$ (2.35), and - 10.76 (2.09) for the lanicemine 50-mg $(n=24)$, $100-\mathrm{mg}(n=24)$, and placebo groups $(n=31)$, respectively.

Experience in conducting clinical trials with putative antidepressant agents delivered parenterally may also have had some effect, as efficacy was similar in sites that participated in both Study 9 and Study 31. MADRS score change from baseline (LS mean $\pm \mathrm{SE}$ ) at week 6 in this subgroup of Study 31 was - 15.97 (13.36), - 19.08 (13.55), and - 13.41 (12.52) for lanicemine 50-mg $(n=37), 100-\mathrm{mg}$ $(n=39)$, and placebo groups $(n=27)$, respectively. Key efficacy outcome measures were comparable across geographical regions.

\section{DISCUSSION}

Adjunctive lanicemine (50 or $100 \mathrm{mg} /$ infusion) provided in 15 doses over a 12-week period was not superior to placebo in reducing depressive symptoms on the primary end point or on any of the secondary measures, at any time point investigated in this study. Both doses of the drug were generally well tolerated and the frequency of AEs and magnitude of psychotomimetic and dissociative effects were consistent with previous studies (Sanacora et al, 2014; Zarate et al, 2013).

The efficacy results stand in contrast to the findings of the previous phase IIb study (Study 9), which showed significant antidepressant effects on similar measures for two doses of lanicemine (100 and $150 \mathrm{mg}$ ) vs placebo over a period of several weeks following nine infusions conducted over 3 weeks (Sanacora et al, 2014). Understanding the reasons for the discrepant findings between Studies 9 and 31 is important, both in evaluating the future value of the NMDAR as a target for antidepressant drug development (Sanacora and Schatzberg, 2015) and as a means of improving study designs for future clinical trials evaluating the efficacy of study drugs delivered under similar conditions.

The first possibility considered is that Study 9 simply produced a false-positive result. Although this is a distinct possibility that cannot be excluded, the magnitude and robustness of the antidepressant effects in that study argue against this conclusion. Differences in study design and subject characteristics between the two studies may potentially have contributed to the differences. The most salient difference between Study 9 to 31 appears to be in the magnitude of placebo response. The current study found a very large, 13.18-point decrease on the MADRS scale with placebo at the 6-week primary end point (vs a 7.9 point decrease at week 3 in Study 9). This 13-point decrease is greater than any reported with active treatment in the three published positive trials with aripiprazole augmentation in similar patients with a history of inadequate response to antidepressant monotherapy (Berman et al, 2007, 2009; Marcus et al, 2008). As it is clear from the existing literature that high placebo response rates were associated with a 

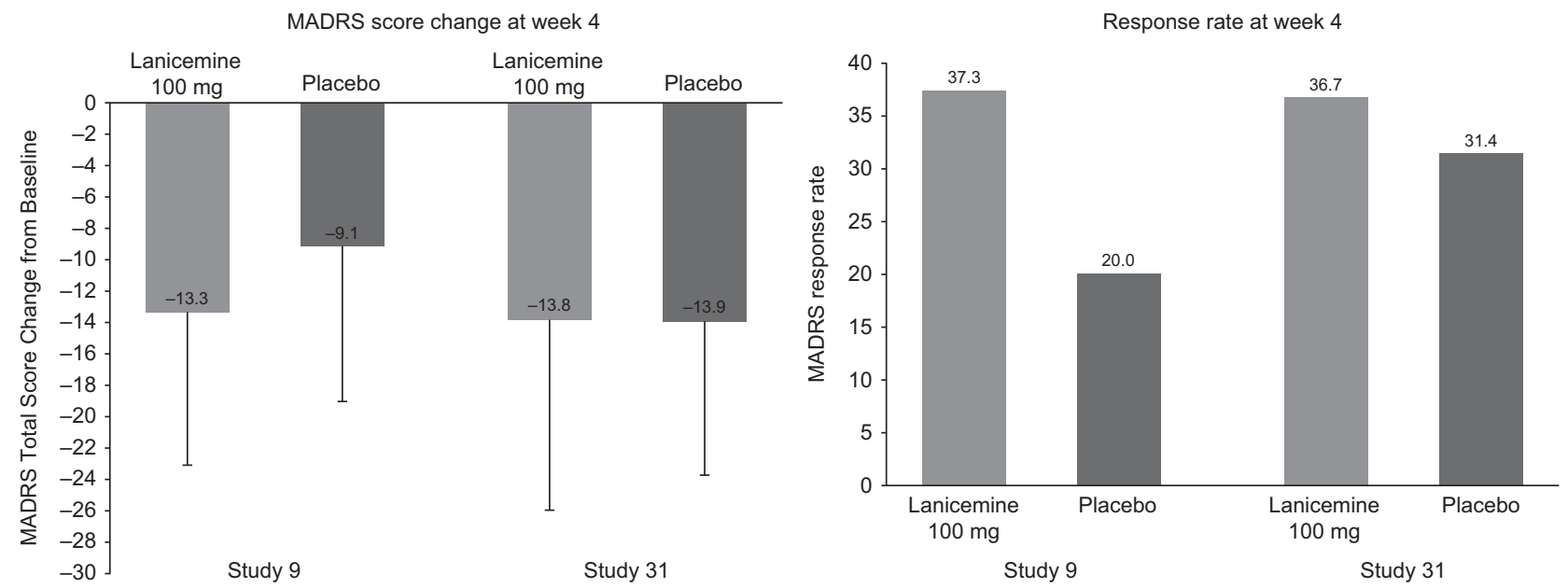

Figure 3 Comparison of efficacy end points in Study 9 and Study 31 in lanicemine $100 \mathrm{mg}$ and placebo groups at 4-week time point: left: mean (SD)

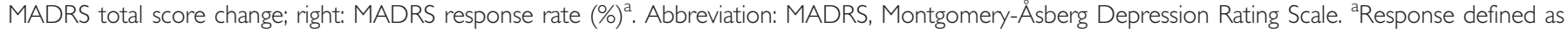
$\geqslant 50 \%$ reduction from baseline in MADRS score.

significantly reduced ability to demonstrate statistical superiority for drug over placebo in phase II and III trials of currently approved antidepressants (Khan et al, 2002a, 2011), there is a distinct possibility that the high placebo response in the current study may have limited the ability to differentiate the drug response from placebo, and thus rendering this a failed trial.

As seen in Supplementary eTable S4, there were several differences in the design of the two phase IIb lanicemine studies that may have contributed to a higher placebo response rate in the current study and the incongruous findings between the studies. Several of these factors, including extended study duration, lack of a placebo runin, and increased number of sites, have previously been shown to influence placebo response in published studies of MDD (Iovieno and Papakostas, 2012; Khan et al, 2002b; Khin et al, 2011; Papakostas et al, 2015). Consistent with reports suggesting a general trend for increased separation between placebo and active antidepressant medications in more severely depressed subjects (Khan et al, 2011; Fournier et al, 2010, 2010; Khin et al, 2011; Naudet et al, 2011), we found evidence that baseline severity impacted drug and placebo responses, with the most severely depressed subjects having the largest antidepressant response to lanicemine $100 \mathrm{mg}$ and the largest separation of drug from placebo. Other limitations of this study include the lack of an assessment of blinding effectiveness, the fact that the required 4-week period on the current antidepressant or drug regimen is relatively short and may have allowed inclusion of patients who would ultimately show response to the ongoing medication, and the lack of information on inter-rater reliability for MADRS scores across sites.

There was a decrease in the frequency of administration of lanicemine or placebo in Study 31 at week 4 (from 3 infusions/week to 1/week) and week 8 (from 1 infusion/week to 1 infusion every other week). By contrast, the dosing schedule in Study 9 was 3 infusions/week throughout the study. The change in frequency of infusion in Study 31 did not appear to have a major impact on response however, as shown by HAM-D score change over time (Figure 2). Most of the change in HAM-D score was apparent in all groups by the end of the 3 infusions/week schedule, and a plateau was reached for all by the end of 1 infusion/week dosing schedule.

In summary, the data from this study show no evidence to support the efficacy of lanicemine augmentation over placebo in treating a nonresponsive major depressive episode. However, comparing the results of the current study with another recently completed phase IIb trial of lanicemine suggests that study design and subject characteristic factors may have impacted treatment outcome, especially placebo response. This highlights the fact that high levels of placebo-response, contrary to what many may have assumed, remains a serious concern even in clinical trials examining treatment efficacy in refractory depression. Considering the high levels of expectation as well as the increased levels of clinician interaction associated with studies utilizing repeated administration of study drugs under highly controlled and medicalized conditions, it may be especially important to optimize study designs to minimize the level of placebo response in future studies conducted under similar conditions.

\section{FUNDING AND DISCLOSURE}

This study was sponsored by AstraZeneca. GS has received consulting fees form Allergan, Alkermes, AstraZeneca, BioHaven Pharmaceuticals, Hoffman La-Roche, Janssen, Merck, Naurex, Servier Pharmaceuticals, Taisho Pharmaceuticals, Teva, and Vistagen therapeutics over the last 24 months. He has also received additional research contracts from AstraZeneca, Bristol-Myers Squibb, Eli Lilly \& Co., Johnson \& Johnson, Hoffman La-Roche, Merck \& Co., Naurex, and Servier over the last 24 months. Free medication was provided to GS for an NIH sponsored study by Sanofi-Aventis. In addition, he holds shares in BioHaven Pharmaceuticals Holding Company and is a co-inventor on a patent 'Glutamate agents in the treatment of mental 
disorders' Patent number: 8778979. MRJ has received research support from Pfizer, Otsuka, AstraZeneca, Forrest, Glaxo SmithKline, Shire, Purdue, Sunovion, Allergan, Alkermes, Lundbeck, Takeda, and Daiichi-Sankyo. AK is the Principal Investigator of over 415 clinical trials sponsored by over 85 pharmaceutical companies and 30 CROs. AK has done no compensated consulting or speaking on their behalf, nor does he own stock in any of these or other pharmaceutical companies. He is not compensated for his role as author. The Northwest Clinical Research Center enrolled 19 patients in the lanicemine trial. SDA has received research grants from AstraZeneca, Pfizer, Amgen, Cephalon, Cerecor, Forest, Lundbeck, Naurex, Otsuka, Takeda, Targacept, Sunovion, and Sanofi-Aventis. RRR has no disclosures. JPS has served as a consultant for Janssen and Servier. MAB has received research support from Otsuka, Forrest, Orexo, AstraZeneca, TauRx Therapeutics, and Sunovion Pharmaceuticals. JMZ has received research support from AstraZeneca, Cyberonics, ElMinda, Forest, Hoffman-LaRoche, Otsuka, Shire, and Takeda. JMZ has served as an Advisor/Consultant to Addvie, Eli Lilly, Lundbeck, Otsuka, PamLab, Shire, and Takeda. LB has received consulting fees from AstraZeneca, Pfizer Pharmaceuticals, Lundbeck, Squibb, and Eli Lilly over the last 24 months. He has also received additional research contracts from AstraZeneca, Takeda, Lundbeck, and Servier over the last 24 months. SJM has served as a consultant to the following companies in the past 24 months: Alkermes, Cerecor, Genentech, Naurex, and Teva. He has received research grants from Janssen Research \& Development, and Otsuka. H-LS, JAP, KHB, MCQ, TMP, and SP were full-time employees of AstraZeneca at the time of the study.

\section{ACKNOWLEDGMENTS}

We thank all the investigators, sites, and patients involved in this study. Bill Wolvey from PAREXEL provided editorial support funded by AstraZeneca. All authors conceived and/ or designed the work that led to the submission, acquired data, and/or played an important role in interpreting the results; drafted or revised the manuscript, and approved the final version. Clinicaltrials.gov identifier NCT01482221 https://clinicaltrials.gov/ct2/show/NCT01482221.

\section{REFERENCES}

Berman RM, Fava M, Thase ME, Trivedi MH, Swanink R, McQuade RD et al (2009). Aripiprazole augmentation in major depressive disorder: a double-blind, placebo-controlled study in patients with inadequate response to antidepressants. CNS Spectr 14: 197-206.

Berman RM, Marcus RN, Swanink R, McQuade RD, Carson WH, Corey-Lisle PK et al (2007). The efficacy and safety of aripiprazole as adjunctive therapy in major depressive disorder: a multicenter, randomized, double-blind, placebo-controlled study. J Clin Psychiatry 68: 843-853.

Bremner JD, Krystal JH, Putnam FW, Southwick SM, Marmar C, Charney DS et al (1998). Measurement of dissociative states with the Clinician-Administered Dissociative States Scale (CADSS). J Trauma Stress 11: 125-136.

Burgdorf J, Zhang XL, Nicholson KL, Balster RL, Leander JD, Stanton PK et al (2013). GLYX-13, a NMDA receptor glycine-site functional partial agonist, induces antidepressant-like effects without ketamine-like side effects. Neuropsychopharmacology 38: 729-742.

Chowdhury GM, Zhang J, Thomas M, Banasr M, Ma X, Pittman B et al (2016). Transiently increased glutamate cycling in rat PFC is associated with rapid onset of antidepressant-like effects. Mol Psychiatry (e-pub ahead of print 12 April 2016; doi:10.1038/ mp.2016.34).

Emnett CM, Eisenman LN, Taylor AM, Izumi Y, Zorumski CF, Mennerick S (2013). Indistinguishable synaptic pharmacodynamics of the N-methyl-D-aspartate receptor channel blockers memantine and ketamine. Mol Pharmacol 84: 935-947.

Fournier JC, DeRubeis RJ, Hollon SD, Dimidjian S, Amsterdam JD, Shelton RC et al (2010). Antidepressant drug effects and depression severity: a patient-level meta-analysis. JAMA 303: 47-53.

Guy W (1976). ECDEU Assessment Manual for Psychopharmacology. National Institute of Mental Health: Rockville, MD.

Iovieno N, Papakostas GI (2012). Correlation between different levels of placebo response rate and clinical trial outcome in major depressive disorder: a meta-analysis. J Clin Psychiatry 73: 1300-1306.

Khan A, Bhat A, Faucett J, Kolts R, Brown WA (2011). Antidepressant-placebo differences in 16 clinical trials over 10 years at a single site: role of baseline severity. Psychopharmacology (Berl) 214: 961-965.

Khan A, Khan S, Brown WA (2002a). Are placebo controls necessary to test new antidepressants and anxiolytics? Int J Neuropsychopharmacol 5: 193-197.

Khan A, Leventhal RM, Khan SR, Brown WA (2002b). Severity of depression and response to antidepressants and placebo: an analysis of the Food and Drug Administration database. J Clin Psychopharmacol 22: 40-45.

Khin NA, Chen YF, Yang Y, Yang P, Laughren TP (2011). Exploratory analyses of efficacy data from major depressive disorder trials submitted to the US Food and Drug Administration in support of new drug applications. J Clin Psychiatry 72: 464-472.

Li N, Lee B, Liu RJ, Banasr M, Dwyer JM, Iwata M et al (2010). mTOR-dependent synapse formation underlies the rapid antidepressant effects of NMDA antagonists. Science 329: 959-964.

Marcus RN, McQuade RD, Carson WH, Hennicken D, Fava M, Simon JS et al (2008). The efficacy and safety of aripiprazole as adjunctive therapy in major depressive disorder: a second multicenter, randomized, double-blind, placebocontrolled study. J Clin Psychopharmacol 28: 156-165.

McGirr A, Berlim MT, Bond DJ, Fleck MP, Yatham LN, Lam RW (2015). A systematic review and meta-analysis of randomized, double-blind, placebo-controlled trials of ketamine in the rapid treatment of major depressive episodes. Psychol Med 45: 693-704.

Naudet F, Maria AS, Falissard B (2011). Antidepressant response in major depressive disorder: a meta-regression comparison of randomized controlled trials and observational studies. PLoS One 6: e20811.

Newport DJ, Carpenter LL, McDonald WM, Potash JB, Tohen M, Nemeroff CB (2015). Ketamine and other NMDA antagonists: early clinical trials and possible mechanisms in depression. Am J Psychiatry 172: 950-966.

Oquendo MA, Baca-Garcia E, Kartachov A, Khait V, Campbell CE, Richards $\mathrm{M}$ et al (2003). A computer algorithm for calculating the adequacy of antidepressant treatment in unipolar and bipolar depression. J Clin Psychiatry 64: 825-833.

Papakostas GI, Ostergaard SD, Iovieno N (2015). The nature of placebo response in clinical studies of major depressive disorder. J Clin Psychiatry 76: 456-466.

Sanacora G, Schatzberg AF (2015). Ketamine: promising path or false prophecy in the development of novel 
therapeutics for mood disorders? Neuropsychopharmacology 40: 259-267.

Sanacora G, Smith MA, Pathak S, Su HL, Boeijinga PH, McCarthy DJ et al (2014). Lanicemine: a low-trapping NMDA channel blocker produces sustained antidepressant efficacy with minimal psychotomimetic adverse effects. Mol Psychiatry 19: 978-985.

Schatzberg AF (2014). A word to the wise about ketamine. Am J Psychiatry 171: 262-264.

Zarate CA Jr, Mathews D, Ibrahim L, Chaves JF, Marquardt C, Ukoh I et al (2013). A randomized trial of a low-trapping nonselective N-methyl-D-aspartate channel blocker in major depression. Biol Psychiatry 74: 257-264.
This work is licensed under a Creative Commons Attribution-NonCommercial-NoDerivs

International License. The images or other third party material in this article are included in the article's Creative Commons license, unless indicated otherwise in the credit line; if the material is not included under the Creative Commons license, users will need to obtain permission from the license holder to reproduce the material. To view a copy of this license, visit http://creativecommons.org/licenses/by-nc-nd/4.0/

(C) The Author(s) 2017

Supplementary Information accompanies the paper on the Neuropsychopharmacology website (http://www.nature.com/npp) 